\title{
Structural Fingerprinting of Nanocrystals in the Transmission Electron Microscope
}

\author{
Sergei Rouvimov ${ }^{1}$, Peter Moeck ${ }^{1}$, and Stavros Nicolopoulos ${ }^{2}$ \\ ${ }^{1}$ Department of Physics, Portland State University, Portland, OR 97207-0751 \\ ${ }^{2}$ NanoMEGAS SPRL, Boulevard Edmond Machterns No 79, Sint Jean Molenbeek, Brussels, \\ B-1080 Belgium; nanomegas.com, info@nanomegas.com
}

Three novel strategies for the structurally identification of nanocrystals in a Transmission Electron Microscope (TEM) are presented [1]. Either a single High-Resolution Transmission Electron Microscopy (HRTEM) image [2] or a single Precession Electron Diffractogram (PED) [3] can be employed. The structural identification information is in both cases collected from an individual nanocrystal. PED from fine-grained crystal powders may also be utilized. Automation of the former two strategies shall lead to statistically significant results on ensembles of nanocrystals. The structural information that can be extracted from a HRTEM image of an approximately 5 to $10 \mathrm{~nm}$ thick nanocrystal is the projected reciprocal lattice geometry, the 2D symmetry, and a few structure factor amplitudes and phase angles. While the structure factor amplitudes suffer from dynamical diffraction effects and are in addition modified by the (not precisely known) contrast-transfer function of the objective lens, the structure factor phase angles are remarkably stable against dynamical diffraction effects and slight crystal misorientations.

Except for the structure factor phase angles, the same "kind" of structural information can be extracted from a PED. The information that can be used for structural fingerprinting is in this case, however, not limited to the resolution of the TEM. (While the symmetry of a Laue zone in a PED is a point group, the 2D symmetry of a HRTEM image is a space group.) Since precession electron diffraction avoids crystal orientations that result in the simultaneous excitation of more than one strong diffracted beam (as much as this is possible by current techniques), quasi-kinematic reflection intensities are obtained for nanocrystals with thicknesses of up to approximately $50 \mathrm{~nm}$ [3]. Simultaneously present reflections in higher order Laue zones and systematic absences in both the higher and the zero order Laue zones may allow for an unambiguous determination of the 3D space group. Comparing kinematical electron diffraction simulations with experimental PEDs allows, therefore, for structure verifications. A universal precession electron diffraction device that can be interfaced to any older or newer mid-voltage TEM was recently commercialized by the NanoMEGAS company".

Searching for structural information that is extracted from HRTEM images or PEDs in databases and matching it with high figures of merit to that of candidate structures shall allow for highly discriminatory identifications of nanocrystals, even without additional chemical information as obtainable in analytical TEMs. As an alternative to the commercial databases, one may use open-access databases [4-7], e.g. Fig. 1, which provide together some 100,000 crystal structure data sets [8].

\section{References}


[1] P. Moeck and P. Fraundorf, Zeits. Kristallogr. 222 (2007) 634, open access at the journal's web pages: http://www.atypon-link.com/OLD/toc/zkri/222/11?cookieSet=1

[2] R. Bjorge, MSc thesis, Portland State University, May 9, 2007; Journal of Dissertation Vol. 1

(2007), open access: http://www.scientificjournals.org/journals2007/j of dissertation.htm [3] P. Moeck and S. Rouvimov, In: Nano Particle Drug Delivery Systems: II Formulation and Characterization, Y. Pathak and D. Thassu (eds.), Informa Health Care, New York, 2009, in

press

[4] http://nanocrystallography.research.pdx.edu/CIF-searchable

[5] http://www.crystallography.net (in France) mirrored at http://nanocrystallography.org (in Oregon, USA), http://cod.ibt.lt (in Lithuania) and http://cod.ensicaen.fr/ (in France); also accessible under a different search surface at http://fireball.phys.wvu.edu/cod// (in West Virginia, USA)

[6] http://rruff.geo.arizona.edu/AMS/amcsd.php

[7] http://crystdb.nims.go.jp

[8] This research was supported by grants from the Oregon Nanoscience and Microtechnologies Institute and Portland State University's Venture Development Fund.

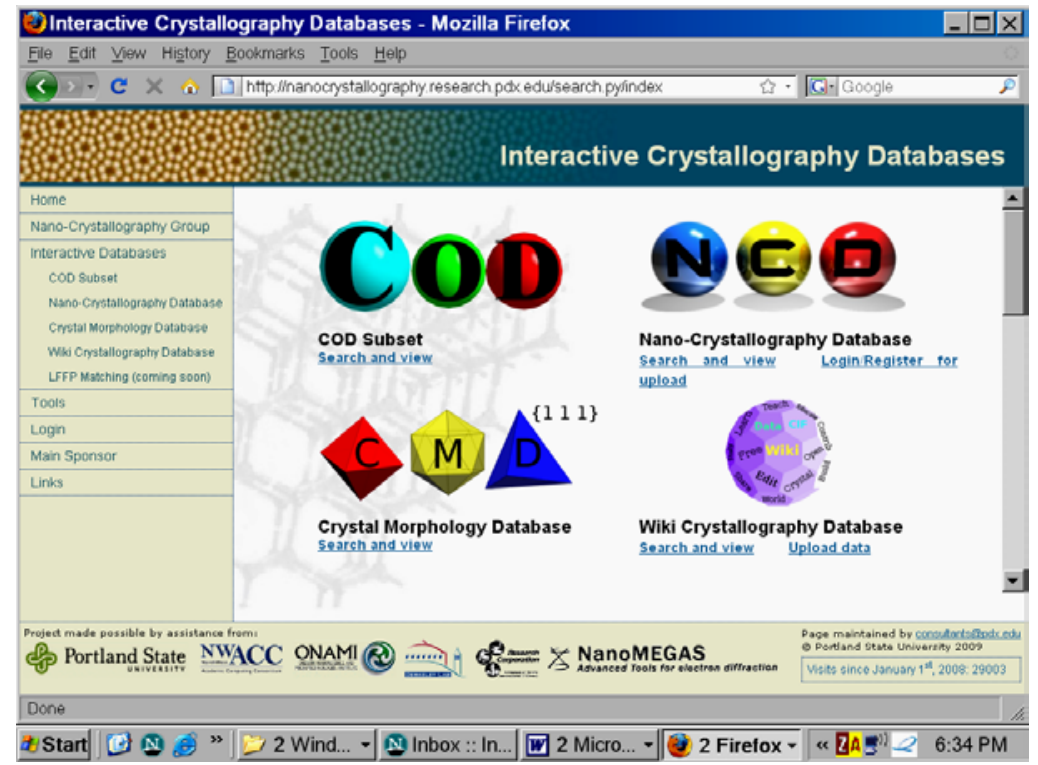

Fig. 1: Access page to approximately 20,000 free datasets with full crystallographic information, i.e.

unit cell dimensions, space group, atomic coordinates, full bibliography [4]. February 7, 2009; note the more than 29,000 hits since January $1^{\text {st }}, 2008$.

"Portland State University's "Laboratory for Structural Fingerprinting and Electron Crystallography" serves as the first demonstration site for the NanoMEGAS company in the Americas. A precession electron diffraction device "Spinning Star" is installed there on an analytical FEI Tecnai $\mathrm{G}^{2}$ F20 field-emission transmission electron microscope and can be demonstrated on request. 